\section{Csk quenches the fire}

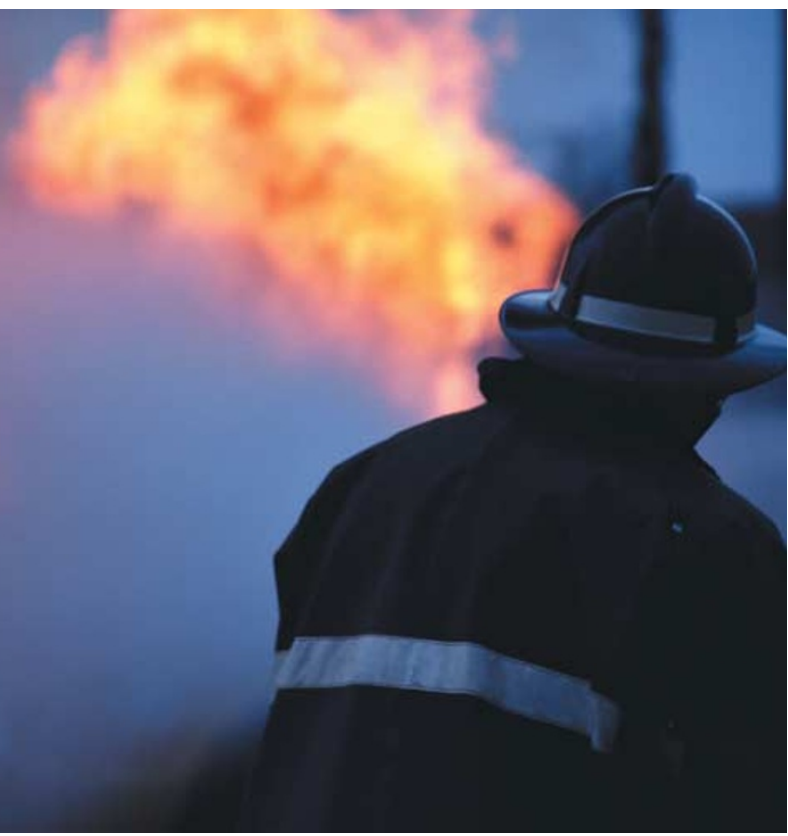

The ligation of integrins on phagocytes triggers a signalling cascade that involves the Src family of tyrosine kinases and is a crucial step in the initiation of inflammatory responses. However, little was known about the regulation of Src-family kinases during inflammation, until a study published recently in Immunity identified carboxy-terminal Src kinase (Csk) as a key negative regulator of these kinases in inflammatory cells.

Csk-deficient mice die in utero. So, to study the role of Csk during acute inflammation, Thomas et al. used a conditional mutagenesis strategy to eliminate Csk in granulocytes (Csk-GEcre). Acute inflammation of the skin and lungs was observed in Csk-GEcre mice housed in conventional facilities, but this was ameliorated by treatment with antibiotics or rederivation of the mice in a specific-pathogen-free environment. Further evidence of a role for Csk in inflammation was indicated by the fact that Csk-GEcre mice showed an enhanced susceptibility to normally sub-lethal doses of lipopolysaccharide, and this was associated with increased serum levels of tumour-necrosis factor.

The hyperresponsiveness of the Csk-GEcre mice correlated with an increased responsiveness of Cskdeficient bone-marrow granulocytes in vitro; culturing in the absence of stimuli induced spontaneous degranulation and upregulation of expression of $\beta_{2}$ and $\beta_{3}$ integrins, as did pharmacological stimulation with PMA and crosslinking of cell-surface integrins. The effect of this increased integrin expression on Csk-deficient granulocyte function was markedly enhanced adhesiveness and decreased chemotaxis, resulting from enhanced actin polymerization and remodelling of the cytoskeleton. Together, these data indicate a negative role for Csk in granulocyte activation.

This increased responsiveness of Csk-deficient bone-marrow granulocytes in vitro was repressed by the inhibitor of Src-family kinases PP2, indicating that hyperactivity in the absence of Csk is mediated, at least in part, by the initiation of

\section{T-CELL ACTIVATION}

\section{How to wake a sleeping lymphocyte}

\author{
According to new research published in \\ Science, downregulation of the Fox \\ (forkhead)-family transcription factor Foxj1 \\ in the lymphoid system can make naive \\ T helper $\left(T_{H}\right)$ cells more responsive \\ to the activating signals that wake them \\ from their slumber. \\ These investigations arose from the initial \\ observation that Foxj1 is downregulated \\ in lymphocytes from mice that are prone \\ to systemic lupus erythematosus. Having \\ shown that Foxj1 is highly expressed by \\ wild-type naive $\mathrm{T}$ cells but is rapidly \\ downregulated when the cells are \\ stimulated, the authors set out to examine \\ the role of this factor in the regulation of \\ immune responses. As most mice with Foxj1 \\ mutations die in utero or soon after birth, \\ they transplanted Foxj $1^{-1-}$ fetal livers into \\ $\mathrm{Rag}^{-1-}$ mice to generate chimeric animals \\ with a Foxj1 $1^{-/}$lymphoid system. Initial \\ analyses showed that these mice had \\ systemic autoimmune inflammation with \\ lymphocytic infiltrates of various organs.
}

T-cell activation is in part controlled by the transcription factor nuclear factor- $\mathrm{KB}$ (NF-KB), and this study went on to show that Foxj1 is an upstream inhibitor of spontaneous and inducible NF- $\kappa \mathrm{B}$ activity in cell lines. Furthermore, Foxj1 $1^{-1-} \mathrm{T}_{\mathrm{H}}$ cells had increased spontaneous NF- $\kappa B$ activity, and blocking NF- $\kappa B$ prevented the hyperresponsiveness of these cells. The inhibitory effect of Foxj1 was probably the result of upregulation of $I \kappa B \beta$, which, as part of the inhibitor of NF- $\kappa \mathrm{B}(\mathrm{I} \kappa \mathrm{B})$ complex, prevents the translocation of NF- $\kappa B$ to the nucleus.

It seems that Foxj1 maintains naive $\mathrm{CD}^{+}$ $\mathrm{T}_{\mathrm{H}}$ cells in a quiescent state by inhibiting NF- $\kappa B$ activity, thereby preventing inappropriate and possibly self-reactive inflammatory responses. This study adds to the known role of other Fox-family members in the immune system, such as Foxp3 in regulatory T cells, Foxo proteins in lymphocyte proliferation and apoptosis, and Foxn 1 in thymic epithelial-cell development.

\section{Kirsty Minton}

(i) References and links ORIGINAL RESEARCH PAPER Lin, L. et al. Modulation of Th1 activation and inflammation by the NF-KB repressor Foxj1. Science 303, 1017-1020 (2004) FURTHER READING Li, Q. \& Verma, I. M. NF-kB regulation in the immune system. Nature Rev. Immunol. 2, 725-735 (2002) 